\section{Minimal-contact psychotherapy for sub-threshold depression in primary care}

\author{
Randomised trial
}

GODELIEF R. W. M. WILLEMSE, FILIP SMIT, PIM CUIJPERS and BEA G. TIEMENS

\begin{abstract}
Background Sub-threshold
depression is a prognostic variable for major depression. Interventions in subthreshold depression may prevent the onset of new cases of major depression.
\end{abstract}

Aims To examine the effects of minimalcontact psychotherapy in primary care patients with sub-threshold depression on the onset of major depression, on the reduction in depressive symptoms and on health-related quality of life.

Method We conducted a randomised trial in primary care, in which patients screened for sub-threshold depression were randomly assigned to minimalcontact psychotherapy $(n=107)$ or to usual care $(n=109)$.

Results One year after baseline, the incidence of major depressive disorder was found to be significantly lower in the psychotherapy group (12\%) than in those receiving usual care (18\%). Small but significant effects were also found on depressive symptoms and on aspects of health-related quality of life.

Conclusions Primarycare patients with sub-threshold depression can benefit from minimal-contact psychotherapy.

Declaration of interest None. Funding detailed in Acknowledgements.
Sub-threshold depression is highly prevalent (Horwarth et al, 1992; Cuijpers et al, 2004); it decreases quality of life (Wells et al, 1992), increases service use (Wagner et al, 2000) and has economic consequences (Broadhead et al, 1990). Furthermore, people with sub-threshold depression have an increased risk of developing major depression (Broadhead et al, 1990; Eaton et al, 1995; Cuijpers et al, 2004). Despite the clinical relevance of this condition, few studies have examined the effects of interventions on subthreshold depression (Muñoz et al, 1995; Barrett et al, 2001; Clarke et al, 2001). In this study we present the results of a trial in which primary care patients with sub-threshold depression were randomised to receive minimal-contact psychotherapy or care as usual. We predicted that the intervention would show superior effects in lowering the incidence of major depression, reducing depressive symptom level and improving health-related quality of life after 1 year.

\section{METHOD}

\section{Study sample}

Study participants were recruited from 19 general practices in The Netherlands. Patients were eligible if they were 18-65 years old and had sub-threshold depression, here defined as having at least one core symptom plus one, two or three current depressive symptoms according to the Instel screening instrument (Tiemens et al, 1995), but not meeting the DSM-IV (American Psychiatric Association, 1994) criteria for full-blown depressive disorder. Exclusion criteria were:

(a) the presence of hearing or language difficulties;

(b) receiving treatment by a mental health professional in the past year, or being on the waiting list for treatment of mental health problems; (c) a diagnosis of a life-threatening illness, learning disability, suicidal risk, psychotic symptoms, schizophrenia or dementia, according to the patient's general practitioner;

(d) meeting DSM-IV criteria for depressive disorder, dysthymia, bipolar disorder, social phobia, agoraphobia or panic disorder in the past 12 months.

\section{Recruitment}

Participants were recruited in two steps (Fig. 1). In the first step, a research assistant approached all patients who were waiting to see their general practitioner $(n=5276)$. The Instel screening questionnaire (see Measures) was filled in during a brief face-to-face interview in a separate room. From the 4525 patients who gave informed consent for screening, 3825 patients were screened. The other 700 patients were excluded on the basis of age or criteria (a) and (b) above. In total, 1018 patients were assessed as having a risk of sub-threshold depression. After office hours, the research assistant consulted the general practitioner to determine if patients who screened positive met exclusion criterion (c). In step two, screen-positive patients who were willing to participate in the trial received a computer-assisted telephone interview with the Composite International Diagnostic Interview (CIDI; World Health Organization, 1997). This resulted in 363 baseline interviews. The main reasons for non-response $(64 \%)$ were lack of interest, lack of time $(47 \%)$ and no telephone contact with the patient $(14 \%$, after at least ten calls at different times and days). In addition, patients who met CIDI/DSM-IV diagnostic criteria for a mood disorder, social phobia, agoraphobia or panic disorder in the past 12 months were excluded $(n=95)$. Patients meeting all inclusion criteria and who gave informed consent were randomised to receive minimal-contact psychotherapy $(n=107)$ or usual care $(n=109)$.

\section{Interventions}

The experimental intervention was minimalcontact cognitive-behavioural therapy for depression, based on the 'Coping with Depression' course (Lewinsohn et al, 1984) in its Dutch version (Cuijpers, 2000). The main component was a self-help manual with instructions on cognitive-behavioural self-help in mood management skills. This intervention has proved to be effective in reducing depressive symptoms in several 


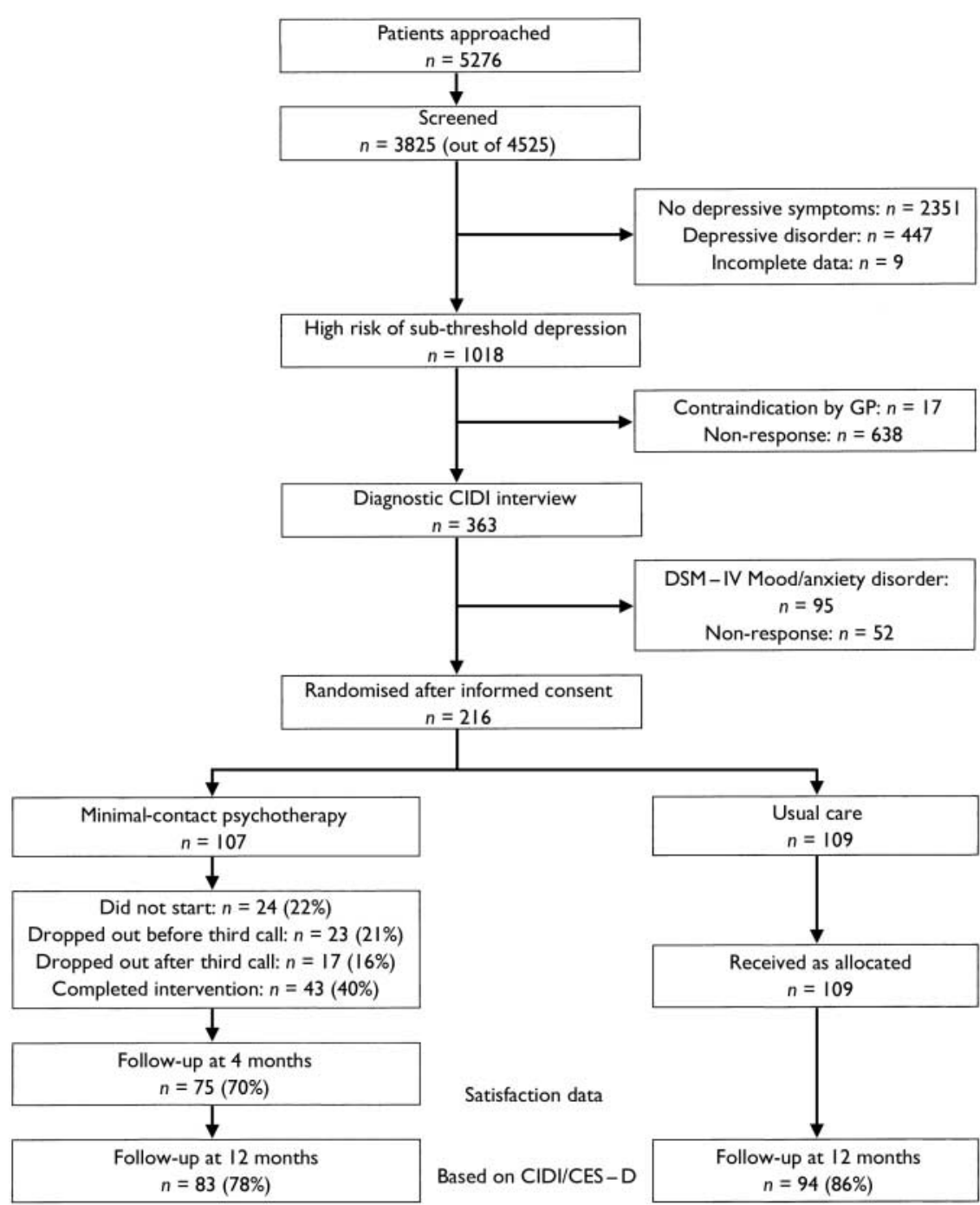

Fig. I Patient flow through the study (CES-D, Center for Epidemiological Studies Depression scale; CIDI, Composite International Diagnostic Interview; GP, general practitioner).

randomised clinical trials, both in group and individual format and in minimalcontact format (Cuijpers, 1998). The manual also included registration exercises and homework assignments. The intervention was augmented by a face-to-face interview with a prevention specialist or a clinician from a community mental health centre before the participant started reading the manual, and six short telephone calls (maximum 15 min each). The first five telephone calls were made once every 2 weeks, and the sixth call was made 2 months later. The telephone contacts were not of a psychotherapeutic nature, but were to support the participants in working through the manual.

Participants receiving the minimalcontact psychotherapy intervention were able to make use of all other types of health services during the intervention period, including those offered by their general practitioner. The comparison (control) intervention was usual care as routinely provided by general practitioners and other health service providers; the former were requested to base their treatments on the Dutch primary care guidelines for depression (Van Marwijk et al, 1994).

\section{Study design}

A power calculation indicated that 200 participants were needed per condition to test the unidirectional hypothesis of a superior effect in the treatment arm of the trial in a one-sided test at $\alpha=0.05$ and a power of $(1-\beta)=0.80$. This sample size would be enough to detect a difference in the incidence rate of $10 \%$ or more (Hully \& Cummings, 1988).

We conducted a pragmatic randomised trial. Eligible participants $(n=216)$ were randomised, with equal probability, to one of the trial arms. The randomisation was carried out centrally, using a blocked randomisation scheme stratified by general practice with the patient as the unit of randomisation, with blocks of four patients.

Data were collected at baseline and at 4 months, 12 months and 24 months after baseline. The 4-month follow-up was meant to collect data on patient satisfaction with the intervention. This paper focuses on the intervention effects at the 12-month follow-up assessment, as the 24-month follow-up data are not yet available. The trial protocol was endorsed by an independent medical ethics committee.

\section{Measures}

Because no (Dutch) screening instrument existed for sub-threshold depression, we used the Instel screen (Tiemens et al, 1995), which was adapted for this purpose. The Instel starts with two core questions about loss of interest and feeling tense, with a negative predictive value of $99 \%$ for either CIDI depressive disorder or generalised anxiety disorder in primary care patients. A depression scale of six symptoms follows after a positive answer on one of the two check questions. For detecting subthreshold depression, the positive predictive values for at least one core symptom plus one, two or three current depressive symptoms were $16 \%, 61 \%$ and $56 \%$, respectively. Negative predictive values were $88 \%, 91 \%$ and $90 \%$, respectively.

The primary outcome of interest is the relative reduction in the incidence of DSM-IV depressive disorder at the 12month assessment, for which the CIDIAuto was used (version 2.1; World Health Organization, 1997) in its Dutch version (Ter Smitten et al, 1998). The CIDI is a standardised diagnostic interview for the assessment of mental disorders, developed by the World Health Organization. It was designed for use by trained lay interviewers. The CIDI is known to have high interrater and test-retest reliability and its validity has been demonstrated to be adequate (Andrews \& Peters, 1998; Wittchen, 1994). The interviews were conducted by telephone, as several findings provide qualified justification for this mode of assessing psychiatric disorder (Rohde et al, 1997; Evans et al, 2004). The interviewers had undergone a prior 3-day training course at the Dutch CIDI training centre. During the data collection, the results of the interviewers were continuously monitored. 
The interviewers were masked with respect to the randomisation status of the participants.

Secondary outcome measures were the number of depressive symptoms and subjective functioning. Depressive symptom level was ascertained with the Center for Epidemiological Studies Depression scale (CES-D; Radloff, 1977) in its Dutch version (Bouma et al, 1995); this is a widely used self-report scale measuring the frequency of 20 depressive symptoms during the past week. The CES-D generates a total score ranging from 0 to 60 , with a higher score indicating more depressive symptoms. The Dutch translation has good reliability and validity (Bouma et al, 1995).

Subjective functioning was measured using the Dutch version of the RAND 36item Health Survey (RAND-36; RAND Health Science Program, 1992; Van der Zee \& Sanderman, 1993), a generic selfreport measure covering eight scales with respect to subjective functioning (see Table 1). Each scale represents a transformed score ranging from 0 to 100 , with a higher score indicating better functioning. The Dutch version has good psychometric properties (Van der Zee \& Sanderman, 1993).

The CES-D data were collected by telephone at baseline and at 12-month and 24month follow-up (together with the CIDI interview). The RAND-36 data were collected at the same measurement points, but using paper and pencil questionnaires, which were sent by post to be completed at home and returned to the research team. The same procedure was followed for the collection of satisfaction data at the 4-month follow-up.

\section{Analysis}

At the 12-month follow-up assessment, $18 \%$ of the CIDI data were missing, mainly owing to the participants' lack of interest. Regression imputation was used to tackle this problem. In the regression imputation model, baseline scores of CES-D, gender and age were used as predictors. Since patients were recruited from 19 general practices some amount of 'clustering' had occurred. This clustering violates the assumption of independence of observations, and may thus affect standard errors and $P$ values. So-called 'robust standard errors' and correct $P$ values were obtained using the first-order Tailor series linearisation method as implemented in
Stata 7.0 (StataCorp, 2001). As there was no significant difference between the two arms of the trial with regard to social and demographic variables and baseline CES-D and RAND-36 scores, it was not necessary to control for confounders.

With regard to the central clinical endpoint, we wanted to test the hypothesis that the incidence rate in the experimental group $\left(I_{\mathrm{E}}\right)$ was lower than the rate in the control group $\left(I_{\mathrm{C}}\right)$ after 1 year; in other words, we tested the hypothesis that the incidence rate ratio $\left(I_{\mathrm{E}} / I_{\mathrm{C}}\right)$ was lower than 1. This was done by regressing the imputed CIDI/DSM-IV depression status at 12 months on the treatment dummy in a Poisson regression while adjusting for the cluster effect.

For the secondary outcomes on continuous measurement scales (CES-D) and RAND-36), last observation carried forward was used to impute missing observations at follow-up. Student's $t$ test (corrected for the cluster effect) was used to test the hypothesis of superior treatment effects in the experimental arm.

All analyses were conducted according to the intention-to-treat principle. The evaluations of the effectiveness were conducted with one-tailed tests, because we were interested in the added value of the intervention therapy in the context of routine primary care. Unless otherwise specified, we used a $5 \%$ significance level $(P<0.05)$. Furthermore, for the CES-D the standardised effect size (d) was calculated by dividing the mean difference of the 12month follow-up score of the control and intervention groups by the 12-month follow-up score standard deviation of the control group (Hedges \& Olkin, 1985).

In order to compare completers and non-completers of the minimal-contact psychotherapy on the level of satisfaction with the intervention, the exact non-parametric Mann-Whitney $U$ test was used.

\section{RESULTS}

\section{Characteristics of the sample}

Socio-demographic characteristics as well as CES-D and RAND-36 baseline scores for each group are presented in Table 1 . No significant difference at $P<0.05$ (or even at $P<0.10$ ) was found between the groups on any of these variables.

Overall, 177 (82\%) people participated in the 12-month follow-up interview. There was no significant difference in follow-up rates between the two trial groups. Completers and non-completers were compared by means of logistic regression analysis on socio-demographic factors and on CES-D and RAND-36 baseline scores. It was found that older people and those with more years of education were more likely to complete the 12-month follow-up interview $\quad(\mathrm{OR}=1.04,95 \%$ CI $1.01-1.08$, $P<0.05 ;$ OR $=1.13,95 \%$ CI $1.00-1.27$, $P<0.05$, respectively). Moreover, men and people with subjectively worse mental health (RAND-36) were less likely to complete the interview $(\mathrm{OR}=0.44,95 \%$ CI $0.20-0.99, P<0.05 ; \quad O R=0.97,95 \%$ CI $0.95-1.00, P<0.05$, respectively).

\section{Effects on the incidence of depressive disorder}

At 12 months, the incidence rate of depressive disorder was $0.12(13 / 107)$ for the minimal-contact psychotherapy condition and $0.18(20 / 109)$ for the usual care condition. The incidence rate ratio was 0.66 (95\% CI 0.40-1.09) and significant in the one-sided test $(P=0.049)$. The number of people to be treated in order to avoid one case of major depression was 16 .

\section{Effects on depressive symptoms and subjective functioning}

The CES-D and RAND-36 scores at baseline and at 12 months are presented in Table 2. Minimal contact psychotherapy was found to have a significant effect on the CES-D and on two scales of the RAND36: 'physical functioning' and 'mental health'. Six of the eight scales of the RAND-36 showed no significant difference. The standardised effect size for the CES-D was 0.18.

\section{Acceptability of the intervention}

Twenty-four (22\%) of the 107 persons randomised to the psychotherapy intervention group refused or failed to start the intervention and another $40(37 \%)$ discontinued it, of whom 23 withdrew before the third telephone call (see Fig. 1). The main reasons for not starting were lack of time $(29 \%)$, not feeling depressed $(21 \%)$ and motivational problems $(17 \%)$. The main reasons for withdrawal were inability to make telephone contact with the individual (23\%), motivational problems $(20 \%)$ and participant not feeling depressed $(20 \%)$.

Completer and non-completer groups were compared by logistic regression 
Table I Baseline characteristics of study participants

\begin{tabular}{lcc}
\hline & Minimal-contact psychotherapy $(n=107)$ & Usual care $(n=109)$ \\
\hline Female, $n$ (\%) & $71(66)$ & $72(66)$ \\
Married/cohabiting, $n$ (\%) & $87(81)$ & $82(75)$ \\
Employed, $n$ (\%) & $84(79)$ & $82(75)$ \\
Age, years: mean (s.d.) & $39.4(11.4)$ & $41.8(11.2)$ \\
Years of education: mean (s.d.) & $14.1(3.6)$ & $13.5(4.2)$ \\
CES-D score: mean (s.d.) & $12.5(8.4)$ & $13.0(8.5)$ \\
Rand-36 score: mean (s.d.): & & $83.4(19.9)$ \\
Physical functioning & $85.8(19.1)$ & $71.8(20.6)$ \\
Social functioning & $72.4(22.7)$ & \\
Role limitations & & $61.4(39.5)$ \\
Physical & $57.8(42.1)$ & $65.0(38.8)$ \\
Emotional & $67.0(38.4)$ & $63.4(17.4)$ \\
Mental health & $66.1(14.2)$ & $53.5(17.8)$ \\
Vitality & $55.1(17.7)$ & $74.0(21.3)$ \\
Pain & $73.5(23.1)$ & $62.2(20.5)$ \\
General health perception & $61.8(19.9)$ & \\
\hline
\end{tabular}

CES-D, Center for Epidemiological Studies Depression scale; RAND-36, RAND 36-Item Health Survey. analysis on socio-demographic factors and on CES-D and RAND-36 baseline scores. It was found that men were less likely to complete the intervention than women $(\mathrm{OR}=0.26,95 \%$ CI $0.10-0.69, P<0.01)$. Furthermore, those who did not complete perceived themselves as having significantly better mental health (RAND-36 score) at baseline compared with those who did complete $(\mathrm{OR}=0.96,95 \%$ CI $0.93-0.99$, $P<0.05$ ).

The 4-month follow-up self-report questionnaire gave information about the satisfaction with the psychotherapy intervention (response rate $70 \%, n=75$ ). In general, participants were satisfied or very satisfied about the coaching from the community mental health centre, the telephone calls (number, length, interval between calls), the homework (difficulty, relevance) and the content of the self-help book. However, participants who discontinued the intervention were significantly less satisfied than those who completed the intervention or who were still working through the intervention at the time of
Table 2 Secondary outcome measures: scores at 12 months

\begin{tabular}{lcccc}
\hline & $\begin{array}{c}\text { Minimal-contact } \\
\text { psychotherapy }(n=107)^{\prime} \\
\text { Mean (s.d.) }\end{array}$ & $\begin{array}{c}\text { Usual care } \\
(n=109)^{\prime} \\
\text { Mean (s.d.) }\end{array}$ & $t$ & $P($ I-sided) \\
\hline CES-D score & $9.4(7.4)$ & $11.1(9.4)$ & 1.98 & 0.032 \\
RAND-36 score: & & & & \\
$\quad$ Physical functioning & $86.7(19.0)$ & $82.5(20.5)$ & -1.77 & 0.047 \\
Social functioning & $77.6(22.2)$ & $74.4(21.3)$ & -0.93 & 0.182 \\
Role limitations & & & & \\
$\quad$ Physical & $69.9(38.3)$ & $65.4(41.3)$ & -0.73 & 0.237 \\
$\quad$ Emotional & $75.1(36.4)$ & $69.5(39.4)$ & -1.13 & 0.137 \\
$\quad$ Mental health & $71.5(14.2)$ & $67.1(18.8)$ & -2.07 & 0.027 \\
$\quad$ Vitality & $57.7(18.5)$ & $55.6(18.0)$ & -1.05 & 0.153 \\
Pain & $77.1(23.5)$ & $73.6(23.9)$ & -0.87 & 0.198 \\
$\quad$ General health perception & $64.5(20.6)$ & $64.2(22.0)$ & -0.10 & 0.462 \\
\hline
\end{tabular}

CES-D, Center for Epidemiological Studies Depression scale; RAND-36, RAND 36 -item Health Survey. I. All analyses were performed on an intention-to-treat base. The group sizes for completers are as follows: psychotherapy group, $n=83$ for CES-D and $n=75$ for RAND-36; usual care group, $n=94$ for CES-D and $n=87$ for RAND-36. completion of the questionnaire. This was true for the coaching from the community mental health centre $(U=201.50, P<0.05)$ and for the experienced relevance of the homework $(U=157.50, P<0.05)$. Also, on several items regarding the perceived effects of the psychotherapy, those who discontinued had scores that were significantly more negative.

\section{DISCUSSION}

\section{Limitations}

This study had several limitations. First, the trial was underpowered, because the number of participants was too small. Notwithstanding its lack of power, the study indicates that the intervention reduced the incidence of depression significantly. Second, the initial response rate was high ( $86 \%$ agreed to be screened), but only $36 \%$ of those identified as having a high risk of sub-threshold depression gave informed consent to participate in the study. Although problems with recruitment and attrition are common in randomised trials of psychological interventions in general practice (Muñoz et al, 1995; Fairhurst \& Dowrick, 1996), this raises questions about the representativeness of the sample. Of the people randomised to the experimental condition, almost $60 \%$ did not start or withdrew from the intervention. On the other hand, most participants $(82 \%)$ did complete the interviews at follow-up and observations that were missing were imputed. All analyses were conducted in accordance with the intention-to-treat principle. Third, this study was conducted in The Netherlands, and the results cannot be generalised safely to primary care systems in other countries. Fourth, it was not possible to mask participants to the condition to which they were assigned; this is true for most randomised trials of psychological interventions, but it may nevertheless have distorted the outcomes of our trial. Because of these limitations, the results of this study should be considered with caution.

\section{Reduction of incidence of major depression}

The incidence of major depression among participants in the experimental condition $(12 \%)$ was reduced by a third compared with the control condition $(18 \%)$. This is an important finding. Two other trials have 
found positive and significant effects of cognitive-behavioural interventions on the incidence of major depressive disorder among adolescents with depressive symptoms (Clarke et al, 1995, 2001). Until now, only one study had examined the possibility of preventing major depression in primary care patients with sub-threshold depression (Muñoz et al, 1995), and this study had insufficient statistical power to find a significant effect. Our trial is therefore the first to show that major depressive disorder can be prevented with a minimal intervention in primary care patients with sub-threshold depression.

Despite this positive finding, we need to address the question of whether we focused this intervention on the right population. An incidence rate of $18 \%$ is considerable, but it is questionable whether it is sufficient to justify specific intervention. After all, more than $80 \%$ of those identified would not develop major depressive disorder within 1 year, and only $6 \%$ would benefit in terms of a prevented episode of major depression. Furthermore, almost $60 \%$ of the patients who were randomised to the experimental condition did not even start the intervention, or failed to finish it. These people perceived their mental health as significantly better at baseline compared with those completing the therapy, and one of the reasons given for withdrawal from the trial was that the person did not feel depressed. The natural course of the symptoms of these patients might have been so positive that an intervention was not necessary.

\section{Effects on depressive symptoms}

The effect of the experimental intervention on depressive symptoms was significant, but relatively small. A reduction in depressive symptoms of 0.18 standard units is usually considered to be small (Lipsey, 1990); psychotherapy and pharmacological treatments for depression usually find reductions of 0.45 standard units or more (Lipsey \& Wilson, 1993) and this is also true for earlier studies of minimalcontact psychotherapy (Cuijpers, 1997). There are several characteristics of our trial that may be responsible for this small effect. First, trial participants were actively recruited by screening, whereas most other trials have recruited people who were seeking care for their depression, through announcements in papers and other media, which might have resulted in a selection of more motivated individuals. Second, most earlier trials of minimal-contact psychotherapy used waiting-list comparison groups, whereas we used a usual care comparison group. Third, previous research has concentrated mostly on people with major depression rather than sub-threshold depression, as tested in this study. Fourth, the small effects might be specific to the group of primary care patients with generally good prognosis. Finally, it is also possible that the small effects are in part caused by the fairly low participation rates in the psychotherapy condition.

\section{Acceptability and suitability of the intervention}

None of the previous trials examining the effects of preventive interventions on the incidence of new cases of depression has made use of minimal-contact psychotherapy. This can be defined as a psychological therapy in which the patient takes home a standardised psychological treatment manual and works through it more or less independently, with only minimal support from professionals. In a metaanalysis of six randomised trials of minimalcontact psychotherapy for depression, it was found that the effects in people with depressive symptoms are large and comparable with the effects of traditional psychotherapy and antidepressive medication (Cuijpers, 1997). This form of therapy is also an interesting intervention for sub-threshold depression in primary care, as it is brief and nonintrusive, and does not require much time or specific skills from the doctor. Because it can be assumed that sub-threshold depression does not justify full psychiatric treatment for a major psychiatric disorder, a minimal intervention aimed at preventing the onset of a depressive disorder seems adequate. The participants in our study who started the intervention and completed a considerable part of it assessed it as positive and helpful. In a systematic review of eight studies examining self-help treatments for anxiety and depressive disorders in primary care (Bower et al, 2001), it was concluded that most studies in this area did find some positive results of these self-help treatments, but also that more research in this area is necessary. Our study adds to the empirical evidence that self-help treatments are beneficial for some patients, but also indicates that more research is needed before this approach can be confidently recommended as an evidence-based practice.

Offering this minimal intervention to a much more selective patient population might increase the effectiveness as well as the efficiency of the intervention. For instance, in a recent trial a preventive intervention was offered to people who not only had sub-threshold depression but also belonged to a high-risk group - adolescent children of depressed parents (Clarke et al, 2001). In this study the incidence of depression dropped from $29 \%$ to $9 \%$. Another possibility for selection is to offer the intervention on a stepped-care base by starting with a short waiting time to exclude patients who recover quickly, because 'watchful waiting' in itself can be an effective strategy (Barrett et al, 2001). Finally, 'open' recruitment strategies should be used in order to reach motivated individuals.

\section{Final remarks}

Depression is one of the most important causes of disability, and is expected to be the second leading cause of disability worldwide in 2020 (Murray \& Lopez, 1996). Reducing the burden of depression is possible, as is shown by the study described here. Perhaps of equal importance is the opportunity to offer a simple self-help treatment, which can be effective while consuming only small amounts of health care resources. The further development and research of preventive interventions in this area constitute a major challenge for prevention science.

\section{ACKNOWLEDGEMENTS}

We thank all patients and general practitioners for taking part in this trial. We also thank the prevention specialists and clinicians from the community mental health centres for coaching the minimal intervention. We acknowledge the input and valuable logistic support of past members of the research team: Fred Leffers, Nynke Terpstra and Simone Onrust. We thank Brigitte Schaefer for supervising the data entry. We acknowledge the contribution of the interviewers in this trial. The study was financially supported by the Healthcare Research Council of The Netherlands (grant 22000026/97-2-268), awarded to P.C.

\section{REFERENCES}

American Psychiatric Association (1994) Diagnostic and Statistical Manual of Mental Disorders (4th edition) (DSM-IV).Washington, DC: APA. 
Andrews, G. \& Peters, L. (1998) The psychometric properties of the Composite International Diagnostic Interview. Social Psychiatry and Psychiatric Epidemiology, 33, 80-88.

Barrett, J. E., Williams, J.W., Oxman, T. E., et al (200I) Treatment of dysthymia and minor depression in primary care: a randomized trial in patients aged 18 to 59 years. Journal of Family Practice, 50, 405-412.

\section{Bouma, J., Ranchor, A.V., Sanderman, R., et a}

(1995) Assessment of Depressive Symptoms with the CESD. Manual (in Dutch). Groningen: Noordelijk Centrum voor Gezondheidsvraagstukken/Rijksuniversiteit Groningen.

Bower, P., Richards, D. \& Lovell, K. (2001) The clinical and cost-effectiveness of self-help treatments for anxiety and depressive disorders in primary care: a systematic review. British Journal of General Practice, 5I 838-845.

Broadhead, W. E., Blazer, D. G., George, L. K., et al (1990) Depression, disability days, and days lost from work in a prospective epidemiological survey. JAMA, 264, 2524-2528.

Clarke, G. N., Hawkins, W., Murphy, M., et al (1995) Targeted prevention of unipolar depressive disorder in an at-risk sample of high school adolescents: a randomized trial of a group cognitive intervention. Journal of the American Academy of Child and Adolescent Psychiatry, 34, 312-321.

Clarke, G. N., Hornbrook, M., Lynch, F., et al (200I) A randomized trial of a group cognitive intervention for preventing depression in adolescent offspring of depressed parents. Archives of General Psychiatry, 58 II27-II34.

Cuijpers, P. (1997) Bibliotherapy in unipolar depression: a meta-analysis. Journal of Behavior Therapy and Experimental Psychiatry, 28, 139-147.

Cuijpers, P. (1998) A psycho-educational approach to the treatment of depression: a meta-analysis of Lewinsohn's 'Coping with Depression' course. Behaviour Therapy, 29, 521-533.

Cuijpers, P. (2000) The Coping with Depression Course (in Dutch). Utrecht/Baarn: Trimbos Institute/Intro.

Cuijpers, P., De Graaf, R. \& Van Dorsselaer, S. (2004) Minor depression: risk profiles, functional disability, health care use and risk of developing major depression. Journal of Affective Disorders, 79, 7I-79.

Eaton, W. W., Badawi, M. \& Melton, B. (1995) Prodromes and precursors: epidemiological data for primary prevention of disorders with slow onset. American Journal of Psychiatry, 152, 967-972.

Evans, M., Kessler, D., Lewis, G., et al (2004) Assessing mental health in primary care research using standardized scales: can it be carried out over the telephone? Psychological Medicine, 34, 157-162.

\section{Fairhurst, K. \& Dowrick, C. (1996) Problems with} recruitment in a randomised controlled trial of counselling in general practice: causes and implications. Journal of Health Services and Research Policy, I, 77-80.

Hedges, L. V. \& Olkin, I. (1985) Statistical Methods for Meta-analysis. Orlando, FL: Academic Press.

Horwarth, E., Johnson, J., Klerman, G. L., et a (1992) Depressive symptoms as relative and attributable risk factors for first-onset major depression. Archives of General Psychiatry, 49, 817-823.

Hully, B. \& Cummings, S. R. (1988) Designing Clinical Research: An Epidemiological Approach. Baltimore, MD: Williams \& Wilkins.

\section{CLINICAL IMPLICATIONS}

- Sub-threshold depression is a clinically relevant condition.

- Minimal-contact psychotherapy may reduce the incidence of major depressive disorder in some primary care patients with sub-threshold depression.

- Minimal-contact psychotherapy may also improve depressive symptoms and aspects of health-related quality of life in people with sub-threshold depression.

\section{LIMITATIONS}

- The statistical power of the trial was relatively small.

- The rate of withdrawal from the intervention was relatively high.

- This study was conducted in The Netherlands, and the results cannot

automatically be generalised to primary care systems in other countries.

GODELIEF R.W. M.WILLEMSE, PhD, FILIP SMIT, MSc, Trimbos Institute/Netherlands Institute of Mental Health and Addiction, Utrecht; PIM CUIJPERS, PhD, Department of Clinical Psychology, Vrije Universiteit Amsterdam, and Trimbos Institute/Netherlands Institute of Mental Health and Addiction, Utrecht; BEA G. TIEMENS, PhD, Gelderse Roos Institute for Professionalizing, Renkum, The Netherlands

Correspondence: Godelief Willemse, Trimbos Institute, PO Box 725, 3500 AS Utrecht, The Netherlands. E-mail: gwillemse@trimbos.nl

(First received 24 October 2003, final revision 16 April 2004, accepted 26 June 2004)

Lewinsohn, P. M., Antonucci, D. O., Breckenridge, J. S., et al (1984) The Coping with Depression Course. Eugene, OR: Castalia.

Lipsey, M.W. (1990) Design Sensitivity: Statistical Power for Experimental Research. Newbury Park, CA: Sage.

Lipsey, M. W. \& Wilson, D. B. (1993) The efficacy of psychological, educational, and behavioral treatment. Confirmation from meta-analysis. American Psychologist 48, $\mid 181-1209$

Muñoz, R. F., Ying, Y., Bernal, G., et al (1995) Prevention of depression with primary care patients: a randomized controlled trial. American journal of Community Psychology, 23, 199-222.

Murray, C. J. \& Lopez, A. D. (1996) The Global Burden of Disease: A Comprehensive Assessment of Mortality and Disability, Injuries and Risk Factors in 1990 and Projected to 2020. Cambridge, MA: Harvard University Press.

Radloff, L. S. (1977) The CES-D scale: a self-report depression scale for research in the general population. Applied Psychological Measurement, I, 385-40I.

RAND Health Science Program (1992) RAND 36 Item Health Survey 1.0. Manual. Santa Monica, CA: RAND.

Rohde, P., Lewinsohn, P. M. \& Seely, J. S. (1997) Comparability of telephone and face-to-face interviews in assessing axis I and II disorders. American Journal of Psychiatry, 154, 1593-1598.

StataCorp (200I) Stata Statistical Software, Release 7.0 College Station, TX: StataPress.

Ter Smitten, M. H., Smeets, R. M.W. \& Van den Brink, W. (1998) Composite International Diagnostic Interview (CIDI), Version 2.I, 12-months (in Dutch). Amsterdam/Geneva: World Health Organization.
Tiemens, B. G., Ormel, J., Brink, R. H. S., van den, et al (1995) Signalering van depressie en gegeneraliseerde angst in de huisartsenpraktijk. De ontwikkeling van een screeningsinstrument. Tijdschrift voor Sociale Gezondheidszorg, 73, 520-527.

Van der Zee, K. I. \& Sanderman, R. (1993) Assessment of General Health with the RAND-36. Manual (in Dutch) Groningen: Noordelijk Centrum voor Gezondheidsvraagstukken/Rijksuniversiteit Groningen.

Van Marwijk, H. W. J., Grundmeijer, H. G. L. M. Brueren, M. M., et al (1994) Dutch general practitioner society Guidelines on Depression (in Dutch). Huisarts en Wetenschap, 37, 482-490.

Van Schaik, D. J. F., Van Marwijk, H.W. J., Van der Windt, D. A.W. M., et al (2002) Effectiveness of psychotherapy for depressive disorder in primary care. A systematic review (in Dutch). Tijdschrift voor Psychiatrie, 44, 609-619.

Wagner, H. R., Burns, B. J., Broadhead, W. E., et a (2000) Minor depression in family practice: functiona morbidity, co-morbidity, service utilization and outcomes. Psychological Medicine, 30, 1377-1390.

Wells, K. B., Burnam, A., Rogers, W., et al (1992) The course of depression in adult outpatients. Archives of General Psychiatry, 49, 788-794.

Wittchen, H.-U. (1994) Reliability and validity studies of the WHO-Composite International Diagnostic Interview (CIDI): a critical review. Journal of Psychiatric Research, 28, 57-84.

World Health Organization (1997) Composite International Diagnostic Interview (CIDI), Version 2.I, 12months. Geneva: WHO. 